\title{
A computational study and meta-analysis of content based image retrieval
}

\author{
Shubham Mathur ${ }^{1 *}$ and Akash Badone ${ }^{2}$ \\ Department of Computer Science and Engineering, Patel College of Science and Technology, Bhopal, Madhya \\ Pradesh, India ${ }^{1}$ \\ Web Developer, SFA Technologies, Bhopal, Madhya Pradesh, India ${ }^{2}$
}

Received: 15-October-2018; Revised: 20-January-2019; Accepted: 23-January-2019

(C)2019 Shubham Mathur and Akash Badone. This is an open access article distributed under the Creative Commons Attribution (CC BY) License, which permits unrestricted use, distribution, and reproduction in any medium, provided the original work is properly cited.

\begin{abstract}
There is the need of efficient image retrieval in different areas including health industry and data processing. It is also important to fetch relevant data for the diagnosis, prediction and data correlation purpose. In the view of the above a study and analysis have been presented for the content based image retrieval (CBIR) methods and their approaches. The objective of this paper is to elaborate and explore the latest trend in this area for the purpose of discovering analytical and computational prospects. So different related and latest proposed and presented methods have been discussed along with the advantages and gaps. Based on the above, the limitations and the problem statements have been highlighted with the suggested solutions.
\end{abstract}

Keywords

Efficient image retrieval, CBIR, Computational analysis, Data correlation.

\section{Introduction}

In the field of image processing there are a number of researchers have been already published different procedures, method and algorithms for the efficient image retrieval. The researchers have proved their potential with different hybridization with the content based image retrieval (CBIR) system [1-5].

This paper main aim is to discuss and provide a computational and analytical view based on these methods and approaches. The current trend indicates the implications of CBIR system as it greatly shows the efficiency in the image retrieval. Due to the extensive demand of this type of system in different areas there is a huge demand of efficient system day by day [6, 7]. There are several methods and approaches in the CBIR system which deals the data retrieval efficiently [8-12].

However, the current scenario shows the demand for the more precise and accurate results as it are also helpful in medical data diagnosis $[13,14]$. So the main objective of this paper is to highlights the problem statements along with the discovery of the possible solutions.

*Author for correspondence

\section{Literature work}

In 2018, Ma et al. [15] presented a new underwater image database. It contains 6240 images from the 40 objects. It is called NWPU underwater image database. They have considered the turbidity value as 0 for the ground-truth. Their method shows that the captured images are closer to the real. For image enhancement they have used multi-scale retinex with color restore (MSRCR) algorithms.

In 2018, Alsabahi et al. [16] discussed the impact of artificial intelligence in case of digital radiography images. They have suggested that the image classification in this case is more difficult than the other normal images. They have used transfer learning which is based on V3 model for the digital radiography images classification.

In 2018, Demirovic et al. [17] suggested that the image processing algorithms suited better in case of parallel architectures multicore central processing unit (CPU) and graphics processing units (GPUs). They have compared relative speedups for different algorithms. They have achieved the speedups range is 3.6 to 15 times. 
In 2018, Altuncu et al. [18] discussed the use of fieldprogrammable gate array (FPGA) in case of unmanned vehicles. They have working in the direction so that the cost can be reduced. They have developed IP core with the functionality of mirroring, inversion, negation, thresholding, brightness and contrast enhancement / reduction on the image. Their results indicate that the hardware cost is low because of their approach.

In 2018, Daniol et al. [19] discussed the use of different image processing applications on the mobile phones. This is also true for the hospital or the medical industry. More and more image processing applications areIntended to run on mobile devices. They have suggested that if the image processing is not efficient then it can affect the performance and the power consumption both negatively. They have compared and analyzed different algorithms used in iPhone. It is based on CPU and GPU. They have considered matrix multiplication, thresholding and Canny's edge detection operations. The results clearly indicates that the GPU based solution can 4 times faster as CPU based.

In 2018, Deperlioglu and Kose [20] discussed regarding the diabetic retinopathy. It is a serious eye disease. They have discussed the applicability of image processing and deep learning in the diagnosis of diabetic retinopathy. Their method is the combination of hue, saturation, value (HSV), V transform algorithm and histogram equalization technics. For the retinal fundus image Gaussian low- pass filter has been used. Classification has been done through the convolutional neural network. The result indicates the efficiency of the approach.

In 2018, Heide et al. [21] discussed and presented a local stereo matching method for hyperspectral camera data. It is used for the multiple usage of camera hardware and for the spectral analysis. The matching method uses the combination of correlation-based similarity. They have evaluated stereo-processing methods. Based on the esult evaluation of CPU and GPU this method has the capability of high speedups.

In 2018, Singh and Arora [22] discuss regarding the biomedical visualization. They have proposed an efficient framework for the abnormal tissue identification in MRI images. They have used texture feature extraction method. They have extracted contrast, correlation, energy, homogeneity and entropy from the gray-level co-occurrence matrix from the tumour images sample. It is inputted to support vector machine. Their approach outperforms in terms of efficiency and accuracy.

In 2019, Li et al. [23] discusses the image superresolution (SR), or the image compactresolution (CR). They have proposed a convolutional neural network (CNN) for image CR. They have named it CNN-CR. It can be trained separately or jointly. Their approach outperforms in terms of reconstruction quality.

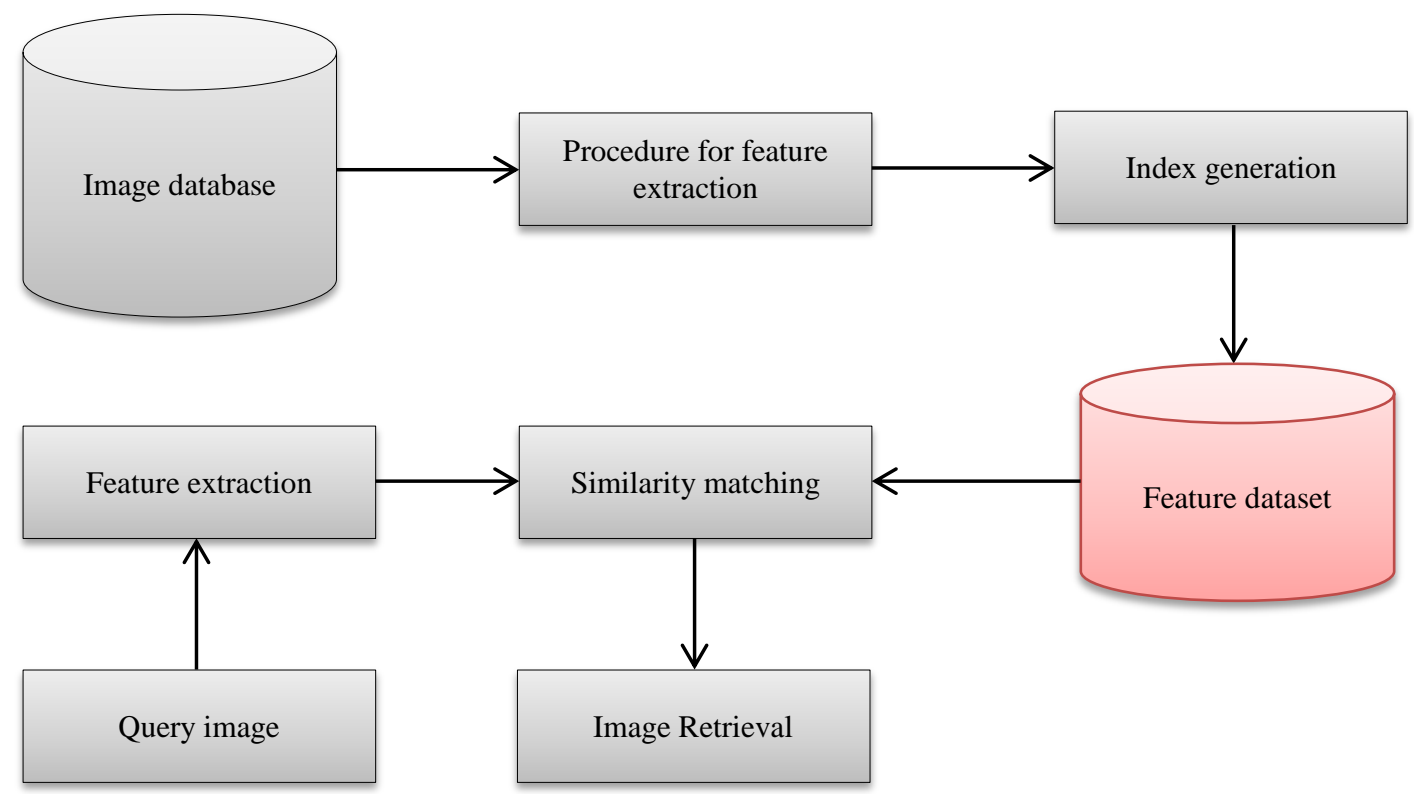

Figure 1 Procedure of content-based image retrieval system 
Figure 1 shows the procedure of content based image retrieval system.

\section{Problem statements}

The study and analysis shows the following problem statements in the previous work:

1. There is the need of image retrieval based on the content as well as the other image features including the matching from the pixel index.

2. There is the need of clustering algorithms for the grouping of the alike properties based on the sub set and super set properties approach.

3. There is the need of higher, lower and average level thresholding for the similarity matching and mapping.
4. Evolutionary algorithms can be important in case of complex attributes and properties.

5. There is the need of hybridization of data filtering, grouping and threshold value mapping so that efficient retrieval is possible.

6. There is the need of the algorithms for the variable dimension and arity.

\section{Study analysis based on method results}

The method analysis based on the methods published earlier is shown in the below Table 1 .

Table 1Comparative analysis based on the results

\begin{tabular}{|c|c|c|c|}
\hline S.No & Reference & Methods & Results achieved \\
\hline 1 & {$[24]$} & $\begin{array}{lr}\text { Dual region } \\
\text { based } & \text { color } \\
\text { channel } & \\
\text { registration } & \end{array}$ & $\begin{array}{l}\text { The results indicates that the method is efficient in generating accurate disparity } \\
\text { map and a high-fidelity color-aligned image. }\end{array}$ \\
\hline 2 & [25] & $\begin{array}{l}\text { Learned } \\
\text { nonlinear local } \\
\text { descriptors }\end{array}$ & $\begin{array}{l}\text { Their method results are as follows: } \\
\text { The mean absolute error of } 75.25 \pm 18.05 \text { Hounsfield units, } \\
\text { Peak signal-to-noise ratio achieved is } 30.87 \pm 1.15 \mathrm{~dB} \text {, } \\
\text { Relative mean absolute error is } 1.56 \pm 0.50 \%\end{array}$ \\
\hline 3 & [26] & $\begin{array}{l}\text { Histopathological } \\
\text { whole slide }\end{array}$ & Their results show the capability of recognizing the malignant regions efficiently. \\
\hline 4 & [27] & $\begin{array}{l}\text { Parallel content- } \\
\text { based image } \\
\text { retrieval system }\end{array}$ & $\begin{array}{l}\text { Their results show that the speedup of the multi-node cluster is more significant than } \\
\text { the single-node cluster. }\end{array}$ \\
\hline 5 & {$[28]$} & $\begin{array}{l}\text { Hybrid features } \\
\text { based CBIR }\end{array}$ & $\begin{array}{l}\text { Their results showthat the better precision accuracy in comparison to the previous } \\
\text { methods. }\end{array}$ \\
\hline 6 & [29] & $\begin{array}{l}\text { CBIR based } \\
\text { colour strings } \\
\text { comparison }\end{array}$ & $\begin{array}{l}\text { Their results based on database classification and colour string coding outperforms as } \\
\text { comparison to the previous methods. }\end{array}$ \\
\hline 7 & {$[30]$} & $\begin{array}{l}\text { Query-sensitive } \\
\text { similarity } \\
\text { measure }\end{array}$ & $\begin{array}{l}\text { The average precision and recall rates are better in comparison to the previous } \\
\text { approaches. }\end{array}$ \\
\hline
\end{tabular}

\section{Conclusion and future scope}

In this paper different aspect of CBIR technique has been analysed and discussed. This paper explores the previous methods with their use and applicability in different areas. It also shows the future enhancements in terms of methods limitations. This paper also discusses the results of some latest works.

The future scopes in this area are as follows:

1. To apply the combination of the mining and soft computing for the better image retrieval with different datasets.
2. To extract the parameters which influence the efficiency and threshold based index calculation for the efficient image retrieval.

3. CBIR techniques can be enhanced with the clustering and classification techniques.

\section{Acknowledgment}

None.

Conflicts of interest

The authors have no conflicts of interest to declare. 


\section{References}

[1] Datta R, Joshi D, Li J, Wang JZ. Image retrieval: ideas, influences, and trends of the new age. ACM Computing Surveys. 2008; 40(2).

[2] Liu Y, Zhang D, Lu G, Ma WY. A survey of contentbased image retrieval with high-level semantics. Pattern Recognition. 2007; 40(1):262-82.

[3] Prasanna MK, Rai SC. Image processing algorithms-a comprehensive study. International Journal of Advanced Computer Research. 2014; 4(15):532-9.

[4] Anandan P, Sabeenian RS. Curvelet based image compression using support vector machine and core vector machine-a review. International Journal of Advanced Computer Research. 2014; 4(15):675-81.

[5] Aggarwal V, Gagandeep. A review: deep learning technique for image classification. ACCENTS Transactions on Image Processing and Computer Vision. 2018; 4(11): 21-5.

[6] Ghosh P, Pandey A, Pati UC. Comparison of different feature detection techniques for image mosaicing. ACCENTS Transactions on Image Processing and Computer Vision. 2015; 1(1):1-7.

[7] Kato T. Database architecture for content-based image retrieval. In image storage and retrieval systems 1992 (pp. 112-24). International Society for Optics and Photonics.

[8] Flickner M, Sawhney H, Niblack W, Ashley J, Huang Q, Dom B, et al. Query by image and video content: the QBIC system. IEEE Computer. 1995; 28(9):23-32.

[9] Pentland A, Picard RW, Sclaroff S. Photobook: content-based manipulation of image databases. International Journal of Computer Vision. 1996; 18(3):233-54.

[10] Mohapatra BN, Panda PP. Histogram equalization and noise removal process for enhancement of image. ACCENTS Transactions on Image Processing and Computer Vision. 2017; 3(9):22-5.

[11] Carson C, Belongie S, Greenspan H, Malik J. Blobworld: image segmentation using expectationmaximization and its application to image querying. IEEE Transactions on Pattern Analysis \& Machine Intelligence. 2002; 24(8):1026-38.

[12] Wang JZ, Li J, Wiederhold G. SIMPLIcity: semanticssensitive integrated matching for picture libraries. IEEE Transactions on Pattern Analysis \& Machine Intelligence. 2001; 23(9):947-63.

[13] Singh H, Kaur T. Novel method for edge detection for gray scale images using $\mathrm{VC}++$ environment. International Journal of Advanced Computer Research. 2013; 3(13):193-7.

[14] Mathur A, Mathur R. Content based image retrieval by multi features using image blocks. International Journal of Advanced Computer Research. 2013; 3(13):251-5.

[15] Ma Y, Feng X, Chao L, Huang D, Xia Z, Jiang X. A new database for evaluating underwater image processing methods. In international conference on image processing theory, tools and applications 2018 (pp. 1-6). IEEE.
[16] Alsabahi YA, Fan L, Feng X. Image classification method in DR image based on transfer learning. In international conference on image processing theory, tools and applications 2018 (pp. 1-4). IEEE.

[17] Demirović D, Skejić E, Šerifović-Trbalić A. Performance of some image processing algorithms in tensorflow. In international conference on systems, signals and image processing 2018 (pp. 1-4). IEEE.

[18] Altuncu MA, Kösten MM, Çavuşlu MA, Şahın S. FPGA-based implementation of basic image processing applications as low-cost IP core. In signal processing and communications applications conference 2018 (pp. 1-4). IEEE.

[19] Daniol M, Boehler L, Keller A, Sroka R. Performance and power consumption evaluation in smartphone based image processing for medical applications. In IEEE international conference on imaging systems and techniques 2018 (pp. 1-5). IEEE.

[20] Deperlığlu Ö, Köse U. Diagnosis of diabetic retinopathy by using image processing and convolutional neural network. In international symposium on multidisciplinary studies and innovative technologies 2018 (pp. 1-5). IEEE.

[21] Heide N, Frese C, Emter T, Petereit J. Real-time hyperspectral stereo processing for the generation of $3 \mathrm{D}$ depth information. In international conference on image processing 2018 (pp. 3299-303). IEEE.

[22] Singh I, Arora M. Image processing based detection of abnormal tissue in magnetic resonance images. In international conference on trends in electronics and informatics 2018 (pp. 1479-83). IEEE.

[23] Li Y, Liu D, Li H, Li L, Li Z, Wu F. Learning a convolutional neural network for image compactresolution. IEEE Transactions on Image Processing. 2019; 28(3):1092-107.

[24] Song S, Park S, Paik J. Dual region based color channel registration for multiple color filtered aperture image. In international conference on electronics, information, and communication 2018 (pp. 1-3). IEEE.

[25] Yang W, Zhong L, Chen Y, Lin L, Lu Z, Liu S, et al. Predicting CT image from MRI data through feature matching with learned nonlinear local descriptors. IEEE Transactions on Medical Imaging. 2018; 37(4):977-87.

[26] Zheng Y, Jiang Z, Zhang H, Xie F, Ma Y, Shi H, et al. Histopathological whole slide image analysis using context-based CBIR. IEEE Transactions on Medical Imaging. 2018; 37(7):1641-52.

[27] Mezzoudj S, Seghir R, Saadna Y. A parallel contentbased image retrieval system using spark and tachyon frameworks. Journal of King Saud UniversityComputer and Information Sciences. 2019:1-9.

[28] Mistry Y, Ingole DT, Ingole MD. Content based image retrieval using hybrid features and various distance metric. Journal of Electrical Systems and Information Technology. 2017; 5: 874-88.

[29] Jenni K, Mandala S, Sunar MS. Content based image retrieval using colour strings comparison. Procedia Computer Science. 2015; 50:374-9. 
[30] Alsmadi MK. Query-sensitive similarity measure for content-based image retrieval using meta-heuristic algorithm. Journal of King Saud University-Computer and Information Sciences. 2018; 30(3):373-81.

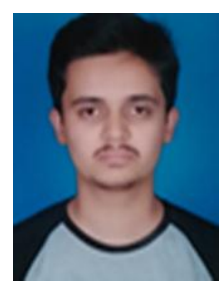

Shubham Mathur has received his Bachelor of Engineering Degree in Computer Science in 2017 from Patel College of Science and Technology, Bhopal, India. His research areas are Image Processing, Computer Vision and Machine Leraning.

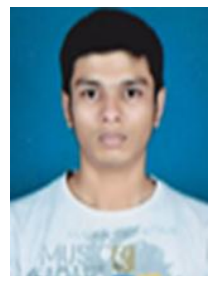

Akash Badone has received his Bachelor of Engineering Degree in Computer Science in 2017 from Patel College of Science and Technology, Bhopal, India. He is currently pursuing M.Tech in Computer Science from Patel College of Science and Technology, Bhopal, India. His research areas are Image Processing, Computer Vision and Machine Leraning.

Email:itseasy21@gmail.com 